\title{
Whole-genome scale identification of methylation markers specific for cerebral palsy in monozygotic discordant twins
}

\author{
ZHE JIAO $^{1}$, ZHIMEI JIANG ${ }^{2}$, JINGTAO WANG ${ }^{3}$, HUI XU ${ }^{3}$, QIANG ZHANG ${ }^{4}$, \\ SHUANG LIU ${ }^{3}$, NING DU ${ }^{4}$, YUANYUAN ZHANG ${ }^{4}$ and HONGBIN QIU ${ }^{1,4}$ \\ ${ }^{1}$ Department of Epidemiology, School of Public Health, Harbin Medical University, Harbin, Heilongjiang 150081; \\ ${ }^{2}$ Heilongjiang Cerebral Palsy Treatment and Management Center; ${ }^{3}$ School of Basic Medicine; \\ ${ }^{4}$ School of Public Health, Jiamusi University, Jiamusi, Heilongjiang 154007, P.R. China
}

Received March 12, 2017; Accepted September 9, 2017

DOI: $10.3892 / \mathrm{mmr} .2017 .7800$

\begin{abstract}
Cerebral palsy (CP) is a severe type of brain disease affecting movement and posture. Although $\mathrm{CP}$ has strong genetic and environmental components, considerable differences in the methylome between monozygotic (MZ) twins discordant for $\mathrm{CP}$ implicates epigenetic contributors as well. In order to determine the differences in methylation in patients with $\mathrm{CP}$ without interference of the interindividual genomic variation, four pairs of $\mathrm{MZ}$ twins discordant for $\mathrm{CP}$ were profiled for DNA methylation changes using reduced representation bisulfite sequencing on the genomic-scale. Similar DNA methylation patterns were observed in all samples. However, MZ twins demonstrated higher correlations and closer evolutionary associations compared with the other samples, indicating a stable methylome of MZ twins. A total of 190 differentially methylated genes (DMGs) were identified using Student's t-test, of which 37 genes were hypermethylated in the $\mathrm{CP}$ group while the remainders were hypomethylated compared with control group. The identified DMGs were enriched in several cerebral abnormalities, including cerebral cortical atrophy and cerebral atrophy, suggesting that the occurrence of $\mathrm{CP}$ may be associated with the methylation alterations. The neighboring genes of DMGs in the protein-protein interaction network were enriched in numerous important functions in essential processes. The results of the present study identified important genes that may epigenetically contribute to the occurrence and development of CP in MZ twins, suggesting that the different prevalence of $\mathrm{CP}$ in identical twins may be associated with DNA methylation alterations.
\end{abstract}

Correspondence to: Professor Hongbin Qiu, School of Public Health, Jiamusi University, 148 Xuefu Street, Jiamusi, Heilongjiang 154007, P.R. China

E-mail: qhbin63@163.com

Key words: cerebral palsy, DNA methylation, monozygotic, differentially methylated genes, protein-protein interaction

\section{Introduction}

Cerebral palsy (CP) is a group of permanent disorders lead to lifelong movement disability and postural dysfunction, due a non-progressive interference, lesion, or abnormality in developing immature brain (1). Nearly half of these disabilities are diagnosed in preterm born children, while full-term born children are diagnosed with a prevalence of 2-2.5/1,000 live birth, this number is several times higher in twins $(2,3)$. The role of genetic contribution to CP has been widely investigated. Several CP candidate mutation genes have been identified by whole-genome exome sequencing (4). Rare copy number variants in $50 \mathrm{CP}$ cases were detected and their frequencies were determined (5). However, the epigenome landscape of $\mathrm{CP}$ in monozygotic (MZ) twins still remains largely unknown.

DNA methylation at the C-5 position of cytosine is a key epigenetic modification that plays critical roles in regulating gene expression during the development of embryos and can be inherited by cell division (6-8). Dysregulation of DNA methylation can lead to many complex diseases including various types of cancer $(9,10)$. DNA methylation undergoes dynamic changes during multiple biological processes and pathological changes (11-13). The identification and functional annotation of different methylome between MZ discordant twins is crucial for understanding the roles of DNA methylation in the $\mathrm{CP}$

Reduced representation bisulfite sequencing (RRBS) was first proposed in 2005 (14), and has been proved as a powerful and cost-effective technology to high-throughout generate the genome-scale DNA methylation profiling at single nucleotide resolution in mammalian cells. Here, we described the comprehensive DNA methylation profiles covering most promoters, $\mathrm{CpG}$ islands of $4 \mathrm{MZ}$ twin pairs discordant for $\mathrm{CP}$ by using RRBS and generated the genomic $\mathrm{CG}, \mathrm{CHG}$ and $\mathrm{CHH}$ contexts $(\mathrm{H}=\mathrm{A}, \mathrm{T}$ or $\mathrm{C})$ methylation patterns. We identified 190 differentially methylated genes (DMGs) that exhibited significant different methylation patterns between $\mathrm{CP}$ and normal healthy samples, these DMGs may closely relate with the CP state. The methylome of $4 \mathrm{MZ}$ discordant twin pairs may be a remarkable resource to explore and understand the epigenetic mechanism underlying the $\mathrm{CP}$ in $\mathrm{MZ}$ twins. 


\section{Materials and methods}

Patients and other family members signed an informed consent form. This study was approved by the Jiamusi University Hospital Ethics Committee.

Patients and DNA samples. The 4 pairs of MZ discordant twins in this study were separately collected from Henan and Liaoning (the Third Affiliated Hospital of Zhengzhou University, Shenyang Children's Hospital Rehabilitation Department). Twin pairs met the following criteria: Birth with no obstetric forceps and no brain trauma, or medication-assisted treatment. The ages of MZ twins were 3 or 4 years at the time of enrollment. The CP diagnosis was confirmed by a pediatric rehabilitation specialist using standard published criteria relating to non-progressive disorders of movement control and posture. The overall phenotypic, clinical characters of the study cohort are shown in Table I.

Blood samples were collected into tubes containing EDTA by skilled nurses at the scene. One milliliter from collection of peripheral blood samples was fixed on the FTA card (Whatman patent product for DNA collection, transportation and storage at room temperature), and submitted to zygosity appraisal institutions to test 20 short tandem repeats (STRs). The pair was identified as MZ twins if all the STRs were the same.

Genomic DNA was then isolated from a Qiagen DNeasy Blood \& Tissue kit (Qiagen Inc., Valencia, CA, USA) from peripheral venous blood leukocytes from 4 pairs of MZ twins discordant for $\mathrm{CP}$, according to the manufacturer's instructions and quantified with PicoGreen. Extracted DNA concentrations were greater than $50 \mathrm{ng} / \mathrm{ml}$, and OD260/280 value between 1.8 and 2.0. All the prepared samples were immediately stored at $-80^{\circ} \mathrm{C}$.

Reduced representation bisulphite sequencing and quality control. After DNA samples were extracted, the restriction enzyme was used to cut the DNA into fixed length fragments. These DNA fragments were added dA at 3'-end, equipped with adapters, then size selected to 40-220 bp, treated with bisulfite, PCR amplified, cloned and then pair-end sequenced with 49 bp read length using Illumina high-throughput sequencing system. After a large amount of raw data was generated, low quality reads with a ratio of $\mathrm{N}$ was greater than $10 \%$ and the proportion of bases for which the quality value was $<20$ was more than $50 \%$ of the entire read. Adapter contamination, and duplication pairs were removed during the process of quality control to get the clean reads.

Mapping RRBS clean reads into reference genome and methyl-cytosines analysis. These clean reads then were mapped into the human reference genome (GRCh37) using BSMAP (15). Only reads uniquely aligned into reference genome and of which 5 restriction sites can be retained to perform analysis. Furthermore, the rate of C-T conversion and alignment were obtained from the BSMAP result. All cytosine sites located on sex chromosomes were removed to eliminate the impact of sex differences. The cytosine site in each uniquely mapped read was reported as methylated or unmethylated status with different patterns $(\mathrm{CpG}, \mathrm{CHG}$, $\mathrm{CHH}$ ). And we calculated the methylation level of each site by the formula, $M /(M+U M)$, with $M$ represents the number of methylated reads and UM representing the number of unmethylated reads.

Phylogenetic tree construction. The phylogenetic tree was constructed to assess the 4 pairs of discordant twins evolutionary patterns for DNA methylation. DNA methylation euclidean distance matrices were calculated using the common $\mathrm{CpG}$ sites with coverage greater or equal to $4 \mathrm{x}$ in all the 8 samples. Based on the minimal evolution algorithm, we applied the 'fastme.bal' function in the R package 'ape' to infer the phylogenetic tree (16).

Screening the DMGs. Among 4 pairs of MZ discordant twins, for each pair, one was suffering from CP patient and the other was normal control. The 8 samples were classified into two groups based on their disease state. First, a Student's t-test was applied for each gene to test whether the methylation of all $\mathrm{CpG}$ sites among the gene in the two groups showed a significant difference. The P-values for multiple-testing correction were further adjusted using the false discovery rate (FDR) method. Second, the methylation level of each gene was qualified by the mean value of its cytosines. For each gene, we calculated the mean difference between every pairs of twins. The gene which was regarded DMG satisfied the following two conditions: The genes met strict controlled thresholds $(\mathrm{P}<0.05)$ and the difference of its methylstion value was more than 0.2 in three couples at least at the same direction would be retained for the following analysis. All these analysis processes were implemented in R language (v3.3.1).

Function enrichment analyses of gene sets and genome regions. The DMGs were imported into Database for Annotation, Visualization and Integrated Discovery (DAVID, v6.8) (17) to perform function enrichment analysis using the default parameters. Annotated Gene Ontology (GO) terms and Kyoto Encyclopedia of Genes and Genomes (KEGG) pathways with P-value $<0.05$ were considered to be statistical significant. The P-value was calculated by hypergeometric test or Fisher's exact test. Moreover, GREAT (v.3.0.0) (18) was used to perform the function enrichment analysis of the genome regions of these differentially methylated gene sets. The annotated human phenotype and disease ontology terms selected were found to be significant by hypergeometric test $(\mathrm{P}<0.05)$.

Protein and protein interaction network construction. The human comprehensive protein-protein network was intergrated from 8 commonly used networks contained in the Biomolecular Interaction Network Database (BIND), the Biological General Repository for Interaction Datasets (BioGRID), the Database of Interacting Proteins (DIP), the Human Protein Reference Database (HPRD), intAct, the Molecular INTeraction database (MINT), the Mammalian PPI Database of the Munich Information Center on Protein Sequences (MIPS), PDZBase (a PPI database for PDZ-domains) and reactome (19). The integrated network was composed of 80,980 edges and 13,361 nodes. The DMGs identified from the sequencing data were set as seed genes, and then mapped into the background PPI network to extract sub PPI network which composed of the seed genes and the genes directly connecting to seed genes. 
Table I. Clinical characters of 4 pairs of monozygotic twins discordant for cerebral palsy.

\begin{tabular}{|c|c|c|c|c|c|c|c|c|}
\hline \multirow[b]{2}{*}{ Sex } & \multicolumn{2}{|c|}{ Sy02010f/Sy02011fep } & \multicolumn{2}{|c|}{$\mathrm{Zz01040m/Zz01041mcp}$} & \multicolumn{2}{|c|}{ Zz01051f/Zz01050fcp } & \multicolumn{2}{|c|}{ Zz01060f/Zz01061fcp } \\
\hline & $\mathrm{F}$ & $\mathrm{F}$ & M & M & $\mathrm{F}$ & $\mathrm{F}$ & $\mathrm{F}$ & $\mathrm{F}$ \\
\hline Age (years) & 3 & 3 & 4 & 4 & 3 & 3 & 3 & 3 \\
\hline Cerebral palsy type & - & Spastic & - & Spastic & - & Spastic & - & Spastic \\
\hline Gestational age (weeks) & 31 & 31 & 28 & 28 & 32 & 32 & 39 & 39 \\
\hline Birth weight (kg) & 1.70 & 1.67 & 1.60 & 1.70 & 3.60 & 2.60 & 2.10 & 2.51 \\
\hline Maternal age (years) & 37 & 37 & 34 & 34 & 30 & 30 & 38 & 38 \\
\hline Paternal age (years) & 38 & 38 & 33 & 33 & 31 & 31 & 37 & 37 \\
\hline
\end{tabular}

F, female; M, male.
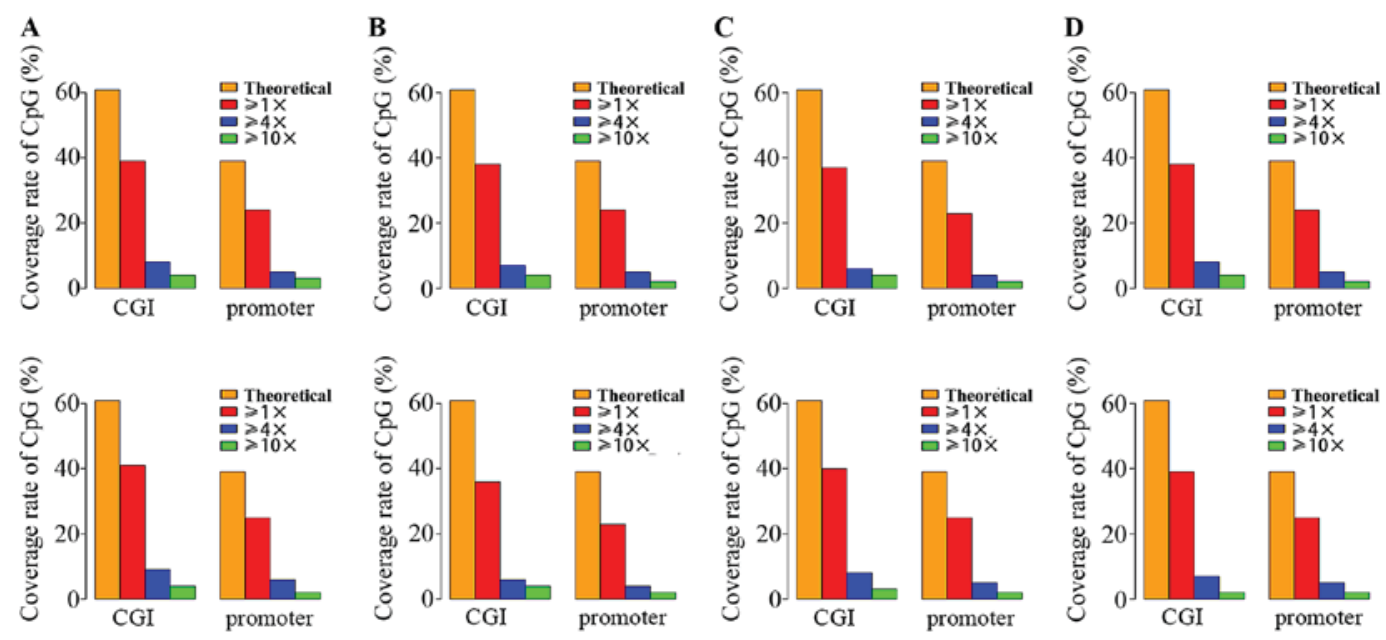

Figure 1. Four discordant twin pairs had similar coverage depth of CpG sites in CGI and promoter regions. (A) Sy02010f/Sy02011fcp, (B) Zz01040m/Zz01041mcp, (C) Zz01051f/Zz01050fcp and (D) Zz01060f/Zz01061fcp. Each image represents one pair of CP twins, respectively. The upper is the control sample and the lower is the CP sample. The yellow bar represents theoretical coverage, which is the ratio of CpG sites located on the theoretical enzyme restriction fragments of promoter/CGI accounting for the total $\mathrm{CpG}$ of the whole genome. The red bar represents the percentage of $\mathrm{CpG}$ loci that were greater than or equal to $1 \mathrm{x}$ related to the total $\mathrm{CpG}$ sites. The blue bar represents the percentage of $\mathrm{CpG}$ loci that were greater than or equal to $4 \mathrm{x}$ related to the total $\mathrm{CpG}$ sites. The green bar represents the percentage of $\mathrm{CpG}$ loci that were greater than or equal to $10 \mathrm{x}$ related to the total $\mathrm{CpG}$ sites. $\mathrm{CP}$, cerebral palsy.

The visualization of the sub-PPI network was performed with cytoscape v3.2.0 (20).

\section{Results}

Description of the $M Z$ twins discordant for $C P$ and RRBS sequencing. The genome-scale identification of the general methylation pattern and different methylome is crucial for us to understand the role of epigenetics in the inconsistent occurrence of $\mathrm{CP}$ in MZ twins, as 4 pairs of MZ CP discordant twins were chosen for RRBS sequencing (Table II). Among the 4 sets of twins, three pairs were female and one was male. The ages ranged from 3 to 4 years. The 8 samples were divided into a disease group and a control group according to their status of CP. DNA extracted from each sample was used for RRBS sequencing to generate a global methylation pattern. The quality process was performed for each sample (see the materials and methods section). A total of 983 million clean reads were retained for these 8 samples. Subsequently, these clean reads were aligned to the reference genome (GRCh37) using the alignment tool BSMAP. There were 718 million reads only uniquely mapped into reference and of which $5^{\prime}$ is restriction sites were obtained to perform the subsequent analysis.

Comprehensive DNA methylation profile of 4 discordant twins pairs. The number of clean reads ranged from 112 to 134 million in the disease group, and 105 to 149 million in the control group. In total, 983 million clean reads from these 8 samples were separately aligned to reference genome using BSMAP. The average uniquely mapped rate between disease group and control group were 73.7 and $72.3 \%$ respectively. By pooling all clean reads together from two groups, 718 million reads were uniquely mapped to the reference genome with the average C-T conversion rate of 99.27\% (Table II). Non-conversion cases were mostly caused by bisulfite treating or sequencing error. The multiple metrics of RRBS sequencing of these 4 pairs of $\mathrm{CP}$ discordant twins have already reached or exceeded anticipated results.

As shown in Fig. 1, the coverage of single nucleotide cytosine of 4 twins at $\mathrm{CpG}$ islands (CGI) was generally higher than promoter regions (Fig. 1). It also can be observed that 


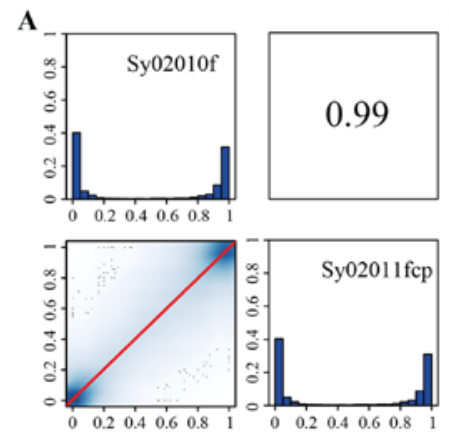

C
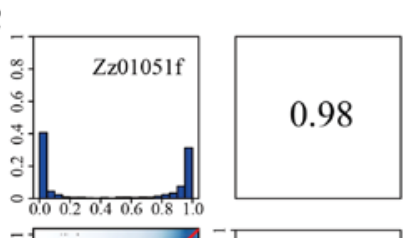

D

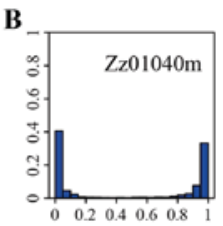

0.99
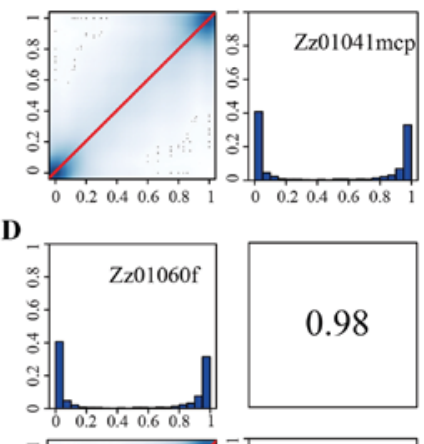

0.98

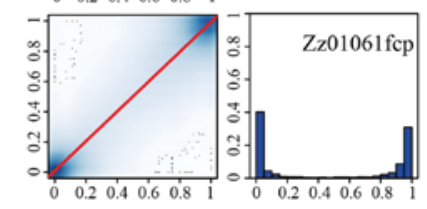

Figure 2. The discordant twin pairs showed high correlation relationship based on whole genome DNA methylation. (A) Sy02010f/ Sy02011fcp, (B) Zz01040m/Zz01041mcp, (C) Zz01051f/Zz01050fcp and (D) Zz01060f/Zz01061fcp represents one pair of CP twins, respectively. The histogram represents the methylation distribution for the twins, of which the title contains 'cp' represents the CP sample and the other is no-CP sample. The smooth scatter represents the methylation correlation between $\mathrm{CP}$ sample and normal, the red line represents the correlation fitting line. $\mathrm{CP}$, cerebral palsy.

there was a difference between the actual single base coverage and the theoretical value according to the RRBS technology. The number of $\mathrm{CpG}$ sites was reduced with the increase of their coverage (14). However, the $\mathrm{CpG}$ sites with high coverage were more credible. Therefore, only the $\mathrm{CpG}$ sites whose coverage $\geq 4$ would be retained to carry out the following analysis.

Correlation of 4 twins discordant with $C P$. It is an extremely rare phenomenon that one of $\mathrm{MZ}$ twins is suffering from $\mathrm{CP}$ and the other is healthy in all 4 pairs in this study. Naturally, it is worthy to explore whether the methylation distribution exhibited significant differences between disease sample and normal sample of each twins. The bimodal distribution of DNA methylation can be observed in each sample of 4 discordant twins pairs (Figs. 2 and 3A), which is consistent with previous knowledge and observations (21). Moreover, the correlations of methylation for each twin pair were $\sim 0.98(\mathrm{P}<0.05)$ (Fig. 2) demonstrating that most of the methylation patterns of MZ twins are consistent, and suggesting the $\mathrm{CP}$ occurrence may be correlated with the DNA methylation alteration on some certain genes/CpG stes.

Simultaneously, the overall methylation level for 8 samples was observed. The methylation level of all samples illustrated the concordant bimodal distribution with the previous studies (21) (Fig. 3A). The methylation level of most CpG sites was 0 (completely unmethylated) or 1 (completely methylated), with few sites showing intermediate methylation levels which may due to the allele-specific methylation sequencing error or 
A

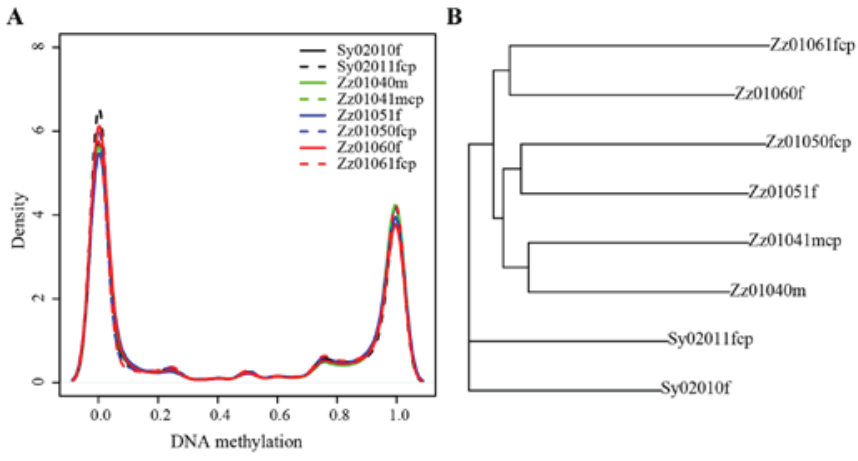

Figure 3. The probability density graph and phylogenetic tree obtained from all CpG sites. (A) All samples exhibited bimodal distribution of their methylation levels in $\mathrm{CpG}$ sites based on the probability density graph. (B) The same MZ twin pairs have more closer evolutionary relationship according to the phylogenetic tree.

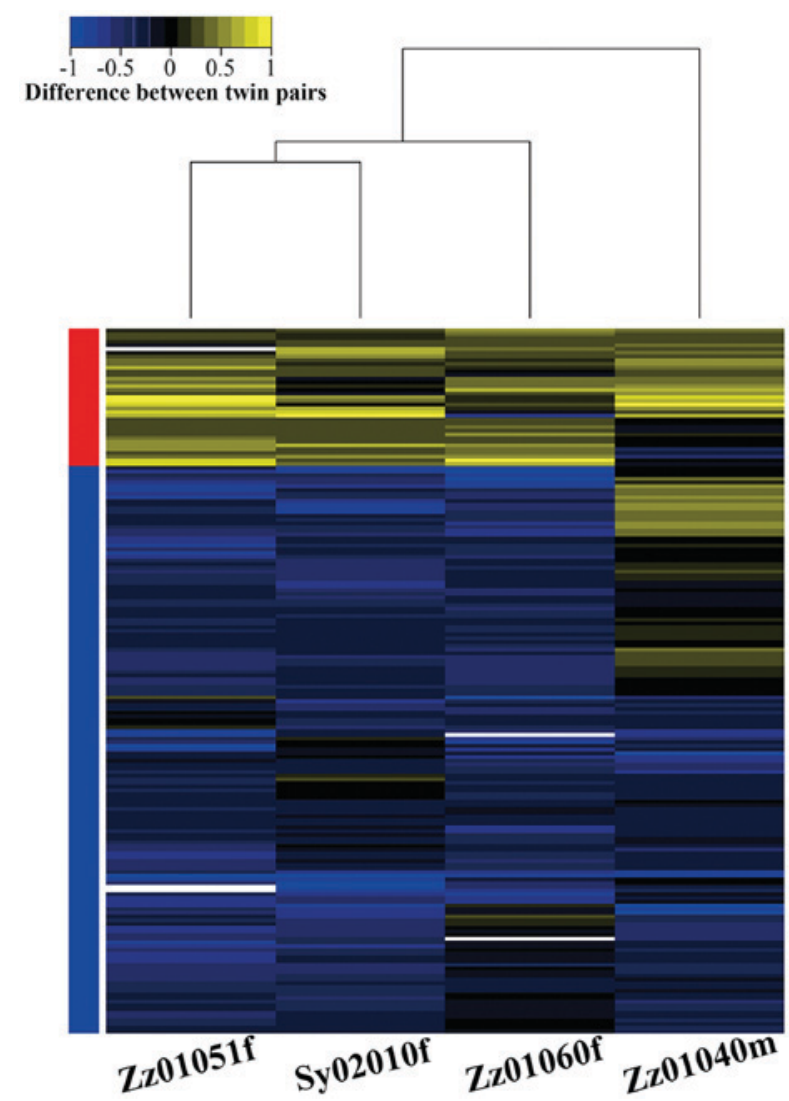

Figure 4. Among the DMGs, 37 genes are hypermethylated in $\mathrm{CP}$ and 153 genes are hypomethylated in CP. Each row represents a DMG, each column represents a pair of twins. The value was the difference between the same-pair twins. Yellow indicates the methylation level in CP is higher than in the control, blue is just on the contrary and black illustrates that there is no difference between the discordant twins. DMGs, differentially methylated genes; CP, cerebral palsy.

the other reasons. Further inferred phylogenetic relationships based on pairwise distances of common $\mathrm{CpG}$ sites among the 4 twins. The phylogenetic tree topology is shown in Fig. 3B. Even though the twins experienced different healthy status, all 4 twins had a closer evolutionary relationship with their twin pair than other families. The phenomenon that the MZ twins shared most of the $\mathrm{CpG}$ site methylation level was expected,

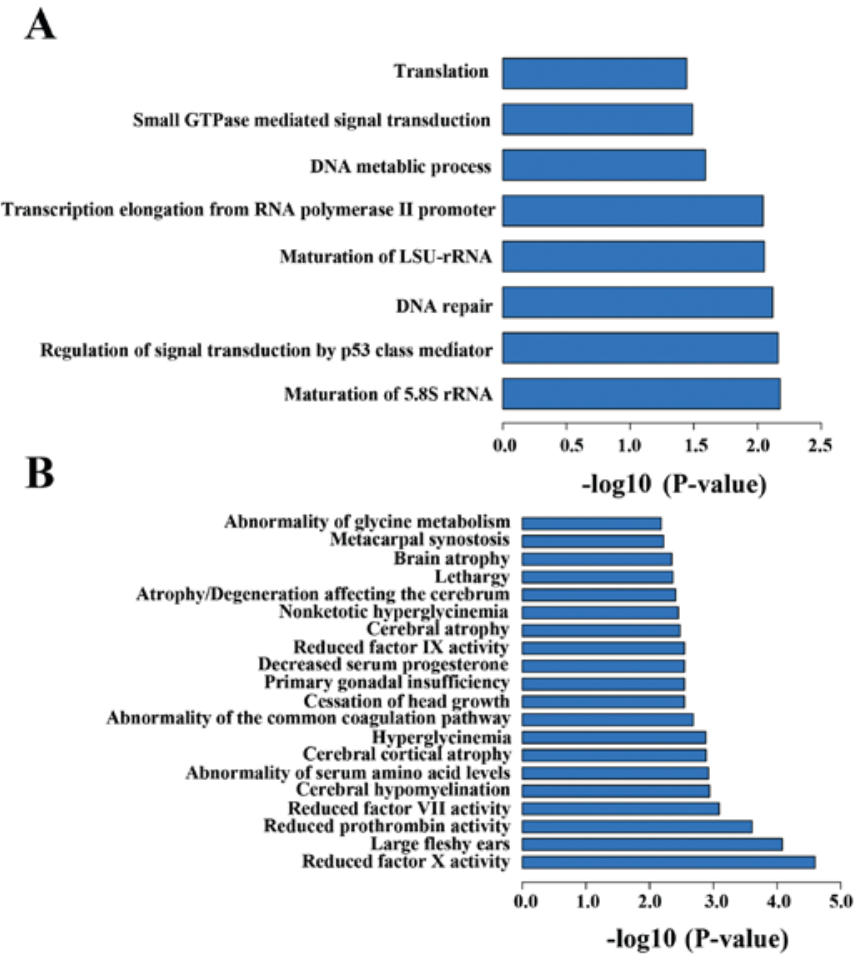

Figure 5. The DMGs were related with several cerebral abnormalities phenotypes. Each blue histogram represents the significantly enriched term, the length was the negative value of the logarithm of the P-value. (A) The biological process enrichment graph of DMGs by DAVID. (B) The phenotype enrichment graph of DMGs by GREAT. Each histogram stands for one disease phenotype. DMGs, differentially methylated genes.

as not all but a few pathogenic sites could drive the occurrence of $\mathrm{CP}$. Herein, the study focused on the excavation of the differentially methylated genes.

The identification of the DMGs. In this study, a total of 190 DMGs were identified by Student's t-test (see Materials and methods). Among them, 37 DMGs showed hypermethylation in the disease group compared to the normal group, and 153 DMGs presented hypomethylation among at least three pairs of twins (Fig. 4). DAVID v6.8 (https://david.ncifcrf.gov/) was used for function enrichment analysis of these 190 DMGs. These genes were mainly enriched in maturation of $5.8 \mathrm{~S}$ rRNA, regulation of signal transduction by $\mathrm{p} 53$ class mediator and some basic biological process like DNA repair, DNA metabolic process (Fig. 5A). These biological process (BP) terms could be disturbed by the abnormal changes in DNA methylation of some pathogenic genes. Moreover, these DMGs were also enriched in biosynthesis of antibiotics, glycolysis/gluconeogenesis, and propanoate metabolism KEGG pathways (Table III). Enrichment analysis for the gene regions of DMGs by GREAT (Fig. 5B) found several cerebral abnormalities such as cerebral cortical atrophy and cerebral atrophy were statistically significant $(\mathrm{P}<0.05)$.

The subnetwork of DMGs from PPI network. It is well known that proteins are involved in almost all physiological processes. To some extent, the PPI network can accurately describe the relationships between proteins. In order to explore the potential functions of the DMGs, 190 DMGs were mapped 
Table III. The enrichment Kyoto Encyclopedia of Genes and Genomes terms.

\begin{tabular}{llcc}
\hline Term & \multicolumn{1}{c}{ Description } & Count & P-value \\
\hline hsa01130 & Biosynthesis of antibiotics & 7 & 0.015 \\
hsa00010 & Glycolysis/gluconeogenesis & 4 & 0.026 \\
hsa00640 & Propanoate metabolism & 3 & 0.029 \\
\hline
\end{tabular}

Table IV. The hub differentially methylated genes among protein-protein interaction network.

\begin{tabular}{llrl}
\hline Ensembl gene ID & Gene symbol & Degree & Direction \\
\hline ENSG00000069869 & NEDD4 & 107 & Down \\
ENSG00000105401 & CDC37 & 74 & Down \\
ENSG00000055332 & EIF2AK2 & 41 & Up \\
ENSG00000114127 & XRN1 & 39 & Down \\
ENSG00000196235 & SUPT5H & 38 & Down \\
ENSG00000126581 & BECN1 & 35 & Up \\
ENSG00000158019 & BRE & 34 & Up \\
ENSG00000070061 & IKBKAP & 33 & Up \\
ENSG00000152467 & ZSCAN1 & 32 & Down \\
ENSG00000103423 & DNAJA3 & 31 & Down \\
\hline
\end{tabular}

NEDD4, neural precursor cell expressed developmentally downregulated protein 4; CDC37, cell division cycle protein; EIF2AK2, eukaryotic translation initiation factor $2 \alpha$ kinase 2; XRN1, 5'-3' exoribonuclease 1; SUPT5H, SPT5 homolog, DSIF elongation factor subunit; BECN1, beclin 1; BRE, brain and reproductive organ-expressed; IKBKAP, inhibitor of $\kappa$ light polypeptide gene enhancer in B-cells, kinase complex-associated protein; ZSCAN1, zinc finger and SCAN domain containing 1; DNAJA3, dnaj heat shock protein family member A3.

into a background network integrated from 8 PPI networks and consisting of 80,980 edges and 13,361 nodes. The DMGs were set as seed genes and the one step neighbors of the DMGs were extracted from the background network. Duplicated relationships were removed, leaving 1,068 nodes and 1,189 edges in the sub-PPI network (Fig. 6). Each node represents a gene and each edge represents the relationship between two nodes. Not all DMGs were present in the sub-PPI network, with only 105 DMGs were retained. Among the network, hub genes were connected with most genes and had higher degrees. These hub genes more tended to perform important functions. Therefore, the top $1 \%$ hub genes in the sub PPI network were identified (Table IV).

In order to find genes which may be truly influenced by the DMGs, we analyzed the network through the network analyzer in cytoscape v3.2.0 and obtained 160 non-differential methylation genes which connected with at least two DMGs simultaneously. These genes were used for performing the enrichment analysis. Amazingly, these genes were associated with many important GO terms (Fig. 7). It is suggested that these DMGs may perform many important features by changing the connected genes through PPI beyond our current understanding. We found that these genes were also enriched in cellular response to nerve growth factor stimulus and that this biological process was related with nervous system which may cause the formation of CP. To a certain extent, the methylation changes of these DMGs may directly or indirectly lead the occurrence of diseases.

\section{Discussion}

Consistent with previous studies, the methylation level in all the four discordant CP pairs exhibited the bimodal distribution in this study. We have observed that each pair of twins exhibited similar methylome profiles, suggesting a stable methylation pattern between MZ twins. We further constructed the phylogenetic tree based on the methylome of these $4 \mathrm{CP}$ pairs of twins. The evolution relationship among the same twins was closer than the others, which indicated the high stability of methylome of MZ twins.

DNA methylation, as one of the most important epigenetic modifications, undergoes dynamic changes during the progress of lesions. A total of 190 DMGs were identified, of which was 37 genes were hypermethylated in CP group and the others were hypomethylated. Surprisingly, functional enrichment of these DMGs has identified several statistically significant human phenotypes closely related with cerebral abnormalities such as cerebral cortical atrophy and cerebral atrophy. The non-DMGs close to the identified DMGs in the comprehensive PPI network were extracted and enriched in multiple basic biological pathways. These genes may be indirectly involved in the occurrence and development of CP.

As twins are the matched controls for nearly all genetic variants and many environmental factors, they provide an opportunity to study DNA methylation (22). CP-discordant MZ twin pairs, who shared an identical DNA sequence, provide an ideal model for examining environmentally driven epigenetic factors in CP. The clusters consist of DMGs and their neighbor non-DMGs in sub-PPI-network identified in this study may provide us with fascinating insights into novel $\mathrm{CP}$ susceptibility genes as well as novel pathogenesis of $\mathrm{CP}$ for drug targeting.

However, there are also some limitations that should be considered when interpreting these results. First, although the study is a genome-wide study of DNA methylation variation in $\mathrm{MZ}$ twin pairs discordant for $\mathrm{CP}$ to date, the sample size for each subgroup (CP and unaffected $\mathrm{CP}$ ) is small, partially sue to the relative rarity of discordant MZ twins. Second, the DNA methylation sequencing was implemented on DNA extracted from peripheral blood rather sample from than the brain. Unfortunately, there is no archived collection of post-mortem brain samples from CP-discordant MZ twins. Nevertheless, recent research suggess that some between-individual epigenetic variation is conserved across brain and blood (23). Third, performing longitudinal studies is the most conclusive approach to disentangle potential cause vs. consequence of DNA methylation changes associated with $\mathrm{CP}$ and is crucial to interpreting the epigenetic effects. The main goal of longitudinal DNA methylation studies is to identify whether DNA methylation changes occur prior to $\mathrm{CP}$ onset and thus may be causal. When possible, longitudinal studies should be a priority 


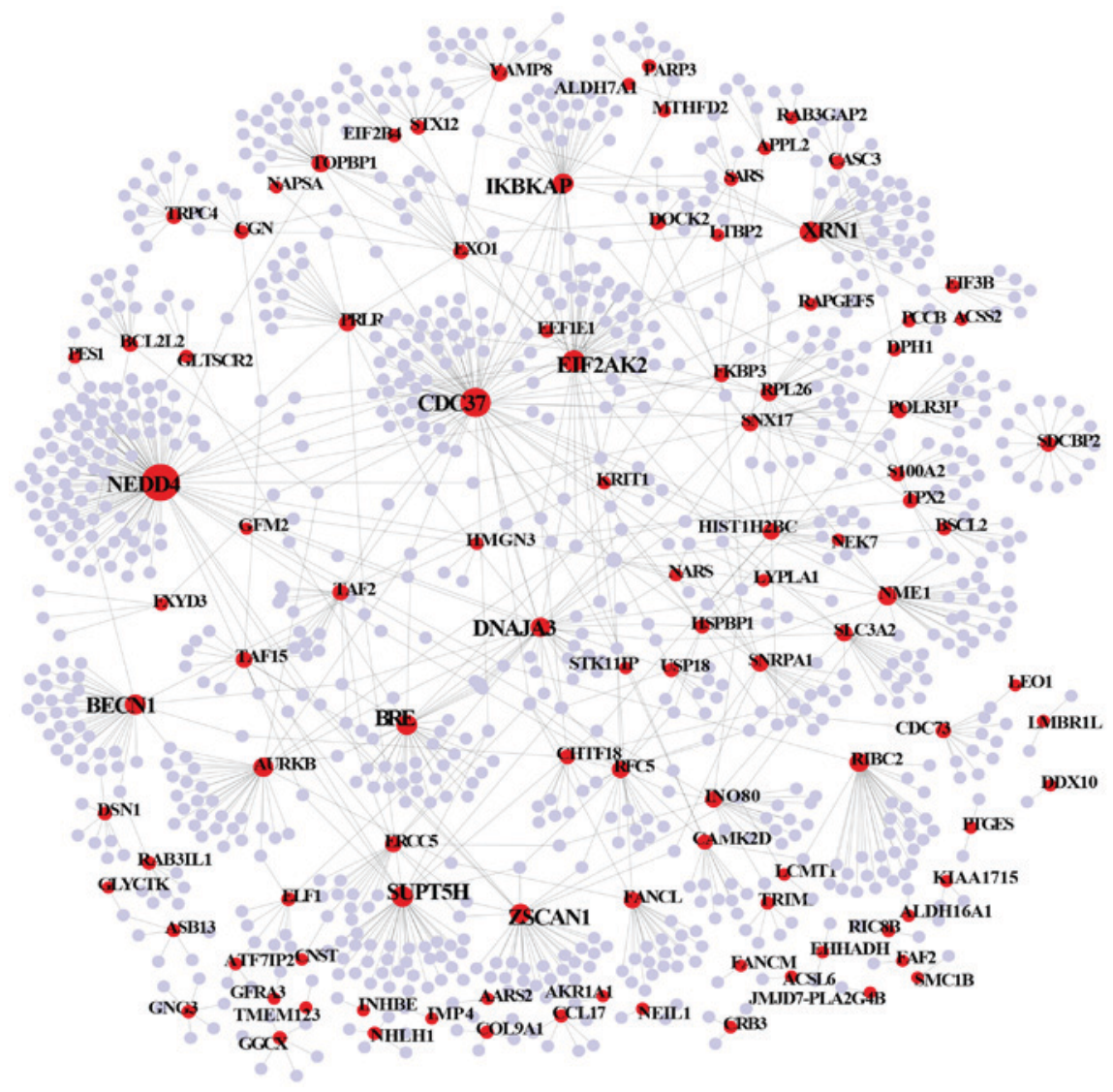

Figure 6. Some DMGs are the hub genes in the sub-PPI network. The sub network extracted from PPI background network is showed. The size of the node changed with the node degree, big node were hub genes always connected with more genes which would prefer to exercise more functions. DMGs, differentially methylated genes.

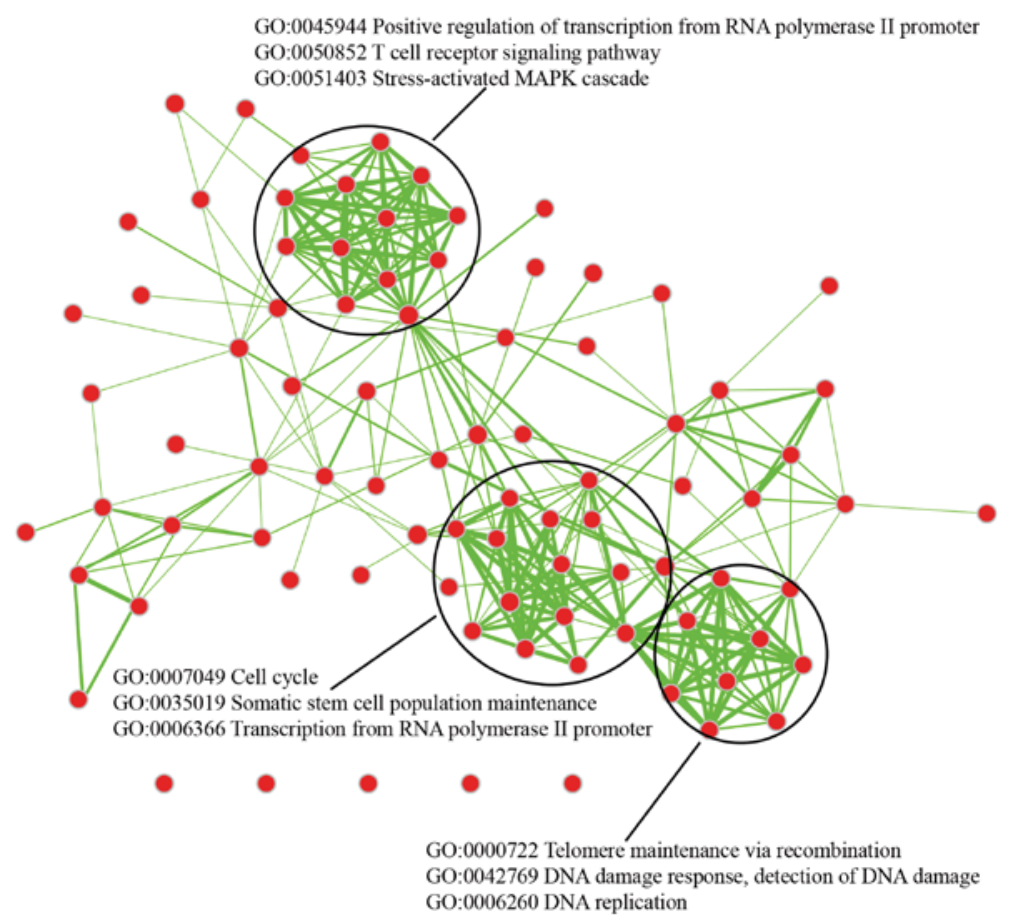

Figure 7. The non-DMGs which were related with at least two DMGs enriched in some basic functions. The nodes represent the gene ontology BP terms from DAVID. The different groups were formed by enrichment map plugin in cytoscape v3.2.0. DMGs, differentially methylated genes.

Finally, it is hard to conclude the causal relationship for any DMGs associated with CP identified in this study because of the lack of the corresponding RNA expression data. Following the collection of more data from CP discordant MZ twins, our 
future study is expected to combine RNA expression and DNA methylation to better explain the aetiology of $\mathrm{CP}$.

In conclusion, the RRBS sequencing of 4 discordant twin pairs at whole-genome scale presented in this study attempts to reveal the methylation landscape of $\mathrm{MZ}$ twins discordant for $\mathrm{CP}$. It should be the first research on the internal links between $\mathrm{CP}$ occurrence and DNA methylation alterations in twins, improving our understanding in the role of methylation in the different disease states in twins. The occurrence of CP is may not only be affected by genetics (24), but also by epigenetic factors.

\section{Acknowledgements}

This study was supported by National Natural Science Foundation of China Project (grant no. 81273174), study on the etiology and pathogenesis of CP based on the twin crowd; and by the Heilongjiang Cerebral Palsy Treatment and Management Center.

\section{References}

1. Palisano R, Rosenbaum P, Walter S, Russell D, Wood E and Galuppi B: Development and reliability of a system to classify gross motor function in children with cerebral palsy. Dev Med Child Neurol 39: 214-223, 1997.

2. Mutch L, Alberman E, Hagberg B, Kodama K and Perat MV: Cerebral palsy epidemiology: Where are we now and where are we going. Dev Med Child Neurol 34: 547-551, 1992.

3. Yokoyama Y, Shimizu T and Hayakawa K: Prevalence of cerebral palsy in twins, triplets and quadruplets. Int J Epidemiol 24: 943-948, 1995.

4. McMichael G, Bainbridge MN, Haan E, Corbett M, Gardner A, Thompson S, van Bon BW, van Eyk CL, Broadbent J, Reynolds C, et al: Whole-exome sequencing points to considerable genetic heterogeneity of cerebral palsy. Mol Psychiatry 20: 176-182, 2015.

5. McMichael G, Girirajan S, Moreno-De-Luca A, Gecz J, Shard C, Nguyen LS, Nicholl J, Gibson C, Haan E, Eichler E, et al: Rare copy number variation in cerebral palsy. Eur J Hum Genet 22: 40-45, 2014

6. Smith ZD and Meissner A: DNA methylation: Roles in mammalian development. Nat Rev Genet 14: 204-220, 2013.

7. Bird A: DNA methylation patterns and epigenetic memory. Genes Dev 16: 6-21, 2002.

8. Wen Y, Wei Y, Zhang S, Li S, Liu H, Wang F, Zhao Y, Zhang D and Zhang Y: Cell subpopulation deconvolution reveals breast cancer heterogeneity based on DNA methylation signature. Brief Bioinform 18: 426-440, 2017.
9. Das PM and Singal R: DNA methylation and cancer. J Clin Oncol 22: 4632-4642, 2004.

10. Timp W and Feinberg AP: Cancer as a dysregulated epigenome allowing cellular growth advantage at the expense of the host. Nat Rev Cancer 13: 497-510, 2013.

11. Liu H, Li S, Wang X, Zhu J, Wei Y, Wang Y, Wen Y, Wang L, Huang Y, Zhang B, et al: DNA methylation dynamics: Identification and functional annotation. Brief Funct Genomics 15: 470-484, 2016

12. Ziller MJ, Gu H, Müller F, Donaghey J, Tsai LT, Kohlbacher O, De Jaqer PL, Rosen ED, Bennett DA, Bernstein BE, et al: Charting a dynamic DNA methylation landscape of the human genome. Nature 500: 477-481, 2013.

13. Varley KE, Gertz J, Bowling KM, Parker SL, Reddy TE, Pauli-Behn F, Cross MK, Williams BA, Stamatoyannopoulos JA, Crawford GE, et al: Dynamic DNA methylation across diverse human cell lines and tissues. Genome Res 23: 555-567, 2013.

14. Meissner A, Gnirke A, Bell GW, Ramsahoye B, Lander ES and Jaenisch R: Reduced representation bisulfite sequencing for comparative high-resolution DNA methylation analysis. Nucleic Acids Res 33: 5868-5877, 2005.

15. Xi Y and Li W: BSMAP: Whole genome bisulfite sequence MAPping program. BMC Bioinformatics 10: 232, 2009.

16. Desper R and Gascuel O: Fast and accurate phylogeny reconstruction algorithms based on the minimum-evolution principle. J Comput Biol 9: 687-705, 2002.

17. National Institute of Allergy and Infectious Diseases (NIAID), NIH: Database for Annotation, Visualization, and Integrated Discovery (DAVID), 2006. https://david.ncifcrf.gov/ease/ease.jsp.

18. McLean CY, Bristor D, Hiller M, Clarke SL, Schaar BT, Lowe CB, Wenger AM and Bejerano G: GREAT improves functional interpretation of cis-regulatory regions. Nature Biotechnol 28: 495-501, 2010.

19. Zhang C, Zhao H, Li J, Liu H, Wang F, Wei Y, Su J, Zhang D, Liu T and Zhang Y: The identification of specific methylation patterns across different cancers. PLoS One 10: e0120361, 2015.

20. Shannon P, Markiel A, Ozier O, Baliga NS, Wang JT, Ramage D, Amin N, Schwikowski B and Ideker T: Cytoscape: A software environment for integrated models of biomolecular interaction networks. Genome Res 13: 2498-2504, 2003.

21. Meissner A, Mikkelsen TS, Gu H, Wernig M, Hanna J, Sivachenko A, Zhang X, Bernstein BE, Nusbaum C, Jaffe DB, et al: Genome-scale DNA methylation maps of pluripotent and differentiated cells. Nature 454: 766-770, 2008.

22. Bell JT and Spector TD: DNA methylation studies using twins: What are they telling us? Genome Biol 13: 172, 2012.

23. Wong CC, Meaburn EL, Ronald A, Price TS, Jeffries AR, Schalkwyk LC, Plomin R and Mill J: Methylomic analysis of monozygotic twins discordant for autism spectrum disorder and related behavioural traits. Mol Psychiatry 19: 495-503, 2013.

24. Nelson KB, Dambrosia JM, Iovannisci DM, Cheng S, Grether JK and Lammer E: Genetic polymorphisms and cerebral palsy in very preterm infants. Pediatr Res 57: 494-499, 2005. 